\title{
Severe emphysematous cystitis: Images in urology
}

\section{Jathin Bandari, MD; Todd Samuel Yecies, MD; Stephen Jackman, MD}

Department of Urology, University of Pittsburgh Medical Center, Pittsburgh, PA

Cite as: Can Urol Assoc J 2015;9(11-12):371. http://dx.doi.org/10.5489/cuai.3254

Published online December 14, 2015

$A^{\prime}$ 78-year-old female with perinuclear anti-neutrophil cytoplasmic antibodies ( $\mathrm{p}-\mathrm{ANCA}$ ) vasculitis on chronic steroids, insulin-dependent diabetes mellitus (HbA1c 6.8), and a history of extended-spectrum beta-lactamase urinary tract infections presented to the emergency room after a ground-level fall. Pelvic computed tomography (CT) scan revealed severe emphysematous cystitis (Fig. 1) with extension into the space of Retzius and through a ventral hernia into subcutaneous fat. She denied dysuria, hematuria, or urinary frequency.

The patient endorsed gradual onset of lower abdominal fullness that has worsened in the days prior to admission. She was initially treated with Foley catheter drainage and meropenem. Urine culture revealed cefuroxime-sensitive Klebsiella pneumonia. She completed a 14-day course of cefuroxime. Repeat CT scan after seven weeks of Foley decompression showed complete resolution (Fig. 2).

Emphysematous cystitis is a rare, but severe infection of the bladder characterized by gas formation within the blad-

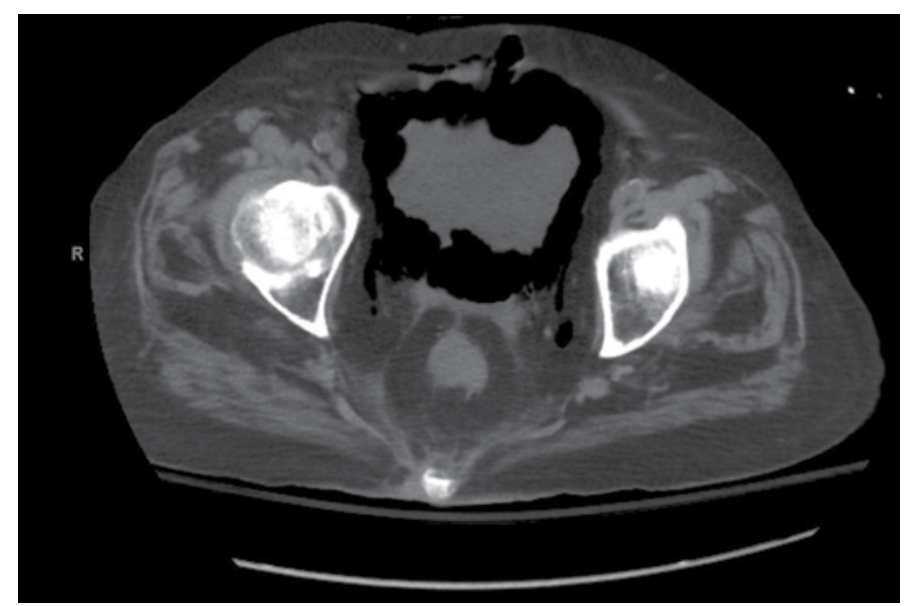

Fig. 1. Representative CT scan images demonstrated diffuse air within the bladder wall extension into the space of Retzius and subcutaneous fat. der wall. It frequently appears dramatic on imaging, but is usually successfully managed with drainage and appropriate antibiotics. ${ }^{1}$ Only $10 \%$ of cases require surgery, with an estimated mortality rate of $7 \% .^{2}$

Competing interests: The authors declare no competing financial or personal interests.

This paper has been peer-reviewed.

\section{References}

1. Quint HJ, Drach GW, Rappaport WD, et al. Emphysematous cystitis: A review of the spectrum of disease. J Urol 1992;147:134-7.

2. Thomas AA, Lane BR, Thomas AZ, et al. Emphysematous cystitis: Review of 135 cases. BJU Int 2007;100:17-20. Epub 2007 May 17. http://dx.doi.org/10.1111/i.1464-410X.2007.06930.x

Correspondence: Dr. Todd Samuel Yecies, Department of Urology, University of Pittsburgh Medical Center, Pittsburgh, PA; yecies@@upmc.edu

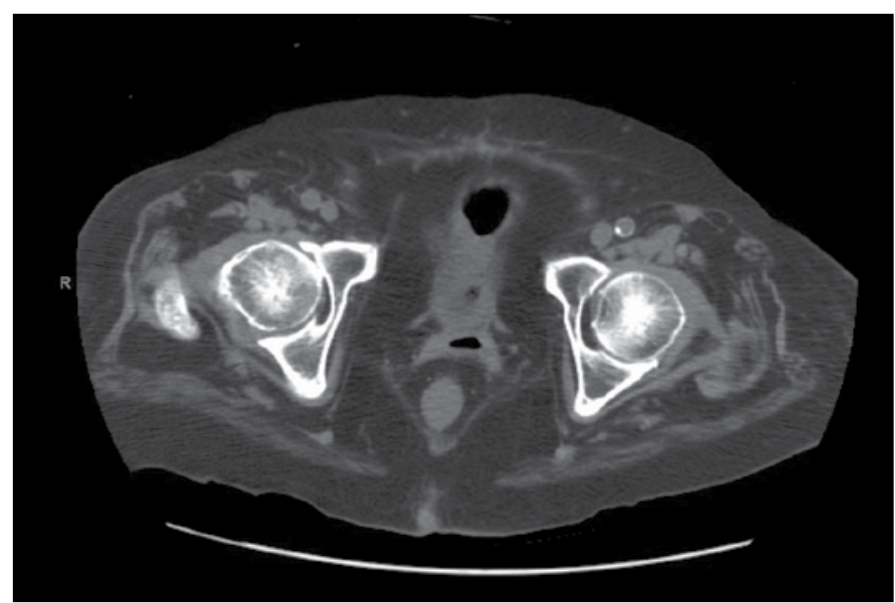

Fig. 2. Repeat CT scan images showing interval resolution of emphysematous cystitis after two weeks of antibiotics and foley catheter drainage. 